\title{
Re-aproximaciones y posibles aplicaciones del concepto de seguridad humana
}

\section{Re-approaches and Possible Applications to the Concept of Human Security}

\author{
Waldimeiry Corrêa da Silva ${ }^{1}$ \\ Universidad Loyola Andalucía (España)
}

Roberta Silva Machado ${ }^{2}$

Universidade Estadual de Campinas (Brasil)

Recibido: 15-03-16

Aprobado: 30-06-16

\section{Resumen}

Este artículo describe y analiza el concepto de seguridad humana en el ámbito de Naciones Unidas y de las Relaciones Internacionales y su relación con la doctrina de la Responsabilidad de proteger (R2P). Pretendemos responder a las siguientes preguntas: ¿La utilización de la doctrina de la Responsabilidad de proteger amplía el concepto de la seguridad humana o lo reduce a su dimensión vertical? ¿El concepto de seguridad humana contribuye a la comprensión y enfrentamiento a la Trata de Personas (la Trata)? Con miras a contestar estas preguntas, el artículo está estructurado en tres partes: en la primera parte, presentamos el marco teóricoconceptual de la seguridad humana con base en los documentos de Naciones Unidas y la teoría de las relaciones internacionales; en la segunda, desde la noción de la R2P, su contenido y práctica internacional, observamos su contribución para el concepto de seguridad humana; en la tercera, utilizamos el enfoque de la seguridad humana para una comprensión global de la Trata.

Palabras-clave: Seguridad humana; Responsabilidad de Proteger; Trata de Personas; Relaciones Internacionales; Naciones Unidas.

\footnotetext{
${ }^{1}$ (wcorrea@uloyola.es) Doctora en Derecho Internacional Público y Relaciones Internacionales por la Universidad de Sevilla (USP-Brasil). Directora del Departamento de Estudios Internacionales de la Universidad Loyola Andalucía. El presente estudio se enmarca dentro del proyecto de investigación: "Las Respuestas del Derecho Internacional y Europeo a los Nuevos Riesgos y Amenazas Contra la Seguridad Humana" (RASEGUR), Plan Nacional de I+D+I (Ref.: DER2015-65906-P) y de la Red de Excelencia sobre "Los actuales desafíos del Derecho Internacional", del Plan Estatal de Investigación Científica y Técnica y de Innovación 2013-2016 (DER15-69273-RED).

2 (robs31@gmail.com) Doctoranda en el Programa de Doctorado en Ciencia Política, área Estudios Internacionales, en el Instituto de Filosofía y Ciencias Humanas de la Universidade Estadual de Campinas (UNICAMP), Brasil. Becaria CAPES, Brasil. Profesora de las Faculdades Campinas (FACAMP), Brasil. 


\begin{abstract}
This article describes and analyzes the concept of human security within the scope of the United Nations and International Relations, and its relationship with the Responsibility to protect doctrine (R2P). We aim to answer the following questions: Does the use of the Responsibility to Protect doctrine widen the concept of human security or reduce it to its vertical dimension? The concepts of human security contribute to the understanding and the fighting against human trafficking? In order to answer these questions, the article is divided into three parts: in the first part, we present the theoretical and historical elements of the concept of human security developed in the United Nations documents, and within the scope of the International Relations Theory; in the second part, we analyze the contribution of the content and international practice of the R2P doctrine to the concept of human security; in the third part, we analyze the concept of human security in order to have a global understanding of human trafficking.
\end{abstract}

Key-words: Human Security; Responsibility to protect; Human trafficking; International Relations, United Nations.

\title{
Introducción
}

El concepto de seguridad humana -y todas sus implicaciones- todavía se encuentra en proceso de evolución. Su propósito es el de alcanzar la realización del desarrollo del ser humano en todas sus dimensiones, logrando con ello la protección de la dignidad humana (Informe de la Comisión de la Seguridad Humana, 2003, p. 6).

A partir de esta aproximación, y llevando en consideración los desafíos que su empleo acarrea desde las posiciones asumidas por los Estados, este artículo tiene por objetivo presentar el concepto de seguridad humana y analizar el alcance de este nuevo paradigma a partir de un breve abordaje histórico y conceptual, de sus aportes y potencialidades a partir del principio de la Responsabilidad de proteger. A pesar de las imprecisiones y discrepancias que permean el tema, tomaremos la reflexión sobre el alcance del paradigma de la seguridad humana, con miras a desenvolver su reflexión relacionada a la Trata de Personas. Adoptamos la seguridad humana como un enfoque alternativo en dos sentidos, uno para describir el marco de la Trata de seres humanos y otro, como discurso político que tiene el potencial para establecer políticas públicas más eficaces y efectivas contra la Trata. Una vez que la perspectiva humanizada de la seguridad permite un aproximación integral y multidisciplinar de la Trata ${ }^{3}$.

${ }^{3}$ En este sentido: "this is a practice often legitimized by a lack of awareness, socio-cultural Araucaria. Revista Iberoamericana de Filosofía, Política y Humanidades, año 18, n 36 . Segundo semestre de 2016. Pp. 217-240. ISSN 1575-6823 e-ISSN 2340-2199 doi: 10.12795/araucaria.2016.i36.10 
A estos efectos, el texto será estructurado en dos partes. En un primer momento, se aborda el marco teórico-conceptual de la seguridad humana, sus fortalezas, debilidades y su prospectiva. De ese modo, recogemos los principales documentos de Naciones Unidas que trataron del tema de la seguridad humana, en especial el Informe del Programa de las Naciones Unidas para el Desarrollo, de 1994, primer documento en el ámbito de la organización que desarrolla el concepto de seguridad humana. Además, analizamos las principales críticas dirigidas al concepto del Informe del PNUD, sobre todo a su dimensión horizontal, es decir, a la vinculación de la seguridad humana con otros aspectos (seguridad medio ambiental, seguridad sanitaria, seguridad alimentaria etc.). Por último, se identificarán el contenido, motivaciones y valores añadidos que la doctrina de la Responsabilidad de proteger ha otorgado al enfoque amplio de la seguridad humana. En la segunda parte, se analizan las contribuciones de la seguridad humana en el enfrentamiento a la Trata de seres humanos. Así, se presenta la política anti-trata en el marco del Protocolo de Palermo y, también, las relaciones entre el concepto de Trata y la seguridad humana, es decir, como la Trata se convierte en un factor que genera inseguridad y requiere acciones eficaces ${ }^{4}$. En las consideraciones finales, se reitera el papel del Estado como garante de la seguridad humana y, consecuentemente, su papel en el combate a la Trata de personas, como también se refuerza la importancia del enfoque de los derechos humanos, reconociendo a las personas como agentes capaces de organizarse con el fin de identificar las amenazas que generan la Trata. Así, se analiza que la seguridad humana, su concepto e implicaciones, y como puede convertirse en una herramienta en el Enfrentamiento a la Trata de Personas.

Se ha realizado una investigación exploratoria y explicativa, cuyo objetivo ha sido el de proporcionar una visión general de la seguridad humana para que se pueda acercar la doctrina de la Responsabilidad de proteger y como esta construcción intelectual podría contribuir a un enfoque integral en torno a la

norms, and lucrative-exploitative economic transactions. Anti-human trafficking solutions require systemic and structural factors linked to poverty, globalization, and political and institutional capacity to protect victims and punish perpetrators. The sustainable human security comprehensive framework provides the necessary framework to study the structural causes of human trafficking while highlighting effective, innovative, and sustainable solutions to the fight against modern human slavery. "(TAVANTI, Marco; TUCKER, Charles E.; WANGERI Gregory J. "Anti-Human Trafficking is at the core of Human Security: An Introduction to the International Journal of Sustainable Human Security Issue on Anti-Human Trafficking". International Journal of Sustainable Human Security (IJSHS). Volume 2, Number 1 (Year 2014-2015): 7-11. www.weinstitute.org/ijshsp.8).

4 "Unfortunately it is a complex phenomenon embedded in many criminal activities and perpetrated by inadequate legal systems, corrupt law enforcement mechanisms and impoverished conditions and fragile populations and social sectors. Indeed, the many global and local human trafficking situations are expressions of a largely unregulated, discriminatory and illegal global marketplace. Honest workers and desperate individuals are lured into job opportunities but exploitative mechanisms for migrant smuggling and indecent labor opportunities in the global supply chain. There is not longer time for complacency as too many people in too many corners of the world are victims and perpetrators of human trafficking". Disponible en: http://www.weinstitute.org/human-trafficking.html. 
Trata de Personas. A estos efectos, hemos realizado una revisión bibliográfica y colecta de datos a través de documentos, bibliografía especializada y otros datos referentes a nuestro objeto de estudio y su posible aplicabilidad en ETP.

\section{El concepto de seguridad humana y contexto de vulnerabilidad}

\subsection{Concepto de seguridad en el pos guerra fría}

El concepto de seguridad humana surgió después de la guerra fría, especialmente como respuesta a las consecuencias graves de los conflictos armados en el inicio de la década de 1990, buscando poner al individuo en el centro de la protección. Al mismo tiempo, empezaron, en las Relaciones Internacionales, nuevos debates respecto de la superación del concepto tradicional de seguridad, basado en la soberanía de los Estados. En este sentido, Buzan y Hansen (2009, p. 12) afirman que, en la pos guerra fría, hubo la necesidad de expandir el concepto de seguridad más allá de la seguridad del Estado. Los estudios para la paz (Peace Research ${ }^{5}$ ) reivindicaron que las necesidades humanas básicas y la violencia estructural tuviesen la misma prioridad en los estudios sobre seguridad.

De ese modo, los estudios sobre seguridad internacional -International Security Studies-empezaron a incluir en sus análisis las dimensiones económica y ambiental de la seguridad. En la década de los noventa del siglo pasado, otras dimensiones empezaron a ser estudiadas: social, desarrollo, salud, género (BUZAN; HANSEN, 2009, p. 12). Tal como señaló Emma Rothishild (1995, p. 55), se produce al menos cuatro tipos de extensión: 1. "E. para bajo" - de la seguridad de los Estados para la de los individuos y grupos, 2. "E. para arriba" - de la seguridad nacional para la seguridad en niveles mucho más amplios como el ambiente/biosfera o la humanidad; 3. "E. horizontal" - de la seguridad militar para la seguridad política, económica, social, ambiental o humana; 4. "E. multidireccional" - de los Estados para las instituciones internacionales, los gobiernos locales o regionales, las organizaciones no-gubernamentales y también la opinión pública, los medios y las fuerzas abstractas de la naturaleza o del mercado. La seguridad humana encaja en esta ola de expansión de la comprensión epistemológica. De acuerdo con Thomas (1987, p. 160), esta

\footnotetext{
${ }^{5}$ Los Estudios para la Paz (Peace Research) surgieron durante el periodo de la guerra fría en oposición a los estudios sobre seguridad con influencia del Realismo Clásico, el cual ponía de manifiesto el equilibrio de poder alcanzado mediante la disuasión nuclear. En la década de 1960, surgieron los primeros institutos sobre los estudios para la paz, como el Peace Research Institute Oslo. Johan Galtung fue uno de los precursores de los abordajes teóricos sobre la paz. En su obra "Violence, Peace, and Peace Research" de 1969 el autor explica que la definición de paz significa ausencia de violencia y esta puede tener distintos componentes, entre los cuales hay la violencia física y la violencia psicológica, y los tres tipos principales de violencia: directa, estructural y cultural.
} 
mirada ha contribuido a replantear el Estado como objeto de referencia de la seguridad, al entender que "son los individuos los que sufren la insatisfacción de las necesidades básicas, constituyendo una amenaza a su seguridad”.

El concepto de seguridad humana está inserido en los estudios críticos sobre seguridad (Critical Security Studies ${ }^{6}$ ), que ponen de manifiesto que las personas deben ser los referentes de la seguridad. A partir de entonces, el Estado tiene un doble rol, es el actor responsable por garantizar seguridad a las personas, pero también puede ser el agente que genera inseguridad (BUZAN; HANSEN, 2009, p. 206). Esta perspectiva humanista (del ser humano en el centro de la protección) recibe un significativo refuerzo del Feminismo ${ }^{7}$, que contribuyó para reforzar la idea de que el individuo debe ser el centro de la protección y, así, contempla la perspectiva de género en sus análisis sobre la seguridad humana. De ese modo, propone que los estudios sobre seguridad internacional traten de temas no contemplados por la visión tradicional (seguridad del Estado), como la violencia contra la mujer en los conflictos armados y las víctimas del tráfico, de la Trata y violencia sexual (BUZAN; HANSEN, 2009, p. 209).

Dicha extensión conceptual de la seguridad humana pone de manifiesto que las amenazas a la seguridad no son solo territoriales, sino que tienen dimensiones económicas, sociales, ambientales etc. Para Buzan y Hansen (2009, p. 203), el concepto recibió críticas por ser demasiado amplio y por poner la seguridad en la agenda de los derechos humanos. Lo que hace relevante la contribución de David Baldwin (1997, pp. 5-26) para comprender los avances del concepto de seguridad humana, es la propuesta de criterios para caracterizar cada enfoque de seguridad, mediante la formulación de cuatro preguntas: ¿Qué es lo que debe ser asegurado? ¿Dónde está la amenaza? ¿Hasta qué punto es extensible el concepto de seguridad? ¿Cuáles amenazas, peligros y urgencias reclaman la seguridad? De ese modo, ¿La Trata de personas es una amenaza a la seguridad? En caso positivo ¿Cómo?

\footnotetext{
${ }^{6}$ Los Critical Security Studies son definidos como Escuela de Frankfurt. En el final de la década de los noventa (fin de la guerra fría), los autores Krause y Williams, en su obra "Critical Security Studies: concepts and cases", de 1997, redefinieron los estudios críticos sobre seguridad, mediante una relectura de los autores de la Escuela de Frankfurt (Teoría crítica), poniendo de manifiesto que los individuos son los referentes de la seguridad y que los Estados promueven más inseguridad que seguridad a los individuos. En este sentido, las discusiones e ideas de los autores de los estudios críticos sobre seguridad se aproximan del concepto de seguridad humana (BUZAN; HANSEN, 2009, pp. 205-206).

${ }^{7}$ El Feminismo en las Relaciones Internacionales es un debate teórico que surge en el final de la guerra fría, junto a otros debates importantes en el área, como la teoría crítica, el posestructuralismo, el constructivismo. El debate feminista hace una crítica al Realismo y a la falta de estudios y debates con perspectiva de género en las Relaciones Internacionales. De ese modo, el abordaje feminista pone de manifiesto que las teorías de relaciones internacionales (Realismo, Liberalismo) no son neutrales y están cargadas de perjuicios, exclusiones y desigualdades, contribuyendo para reforzar una visión occidental y masculina de las relaciones internacionales (RUIZ-GIMÉNEZ ARRIETA, 2000).
} 
Sin embargo, el debate sobre la seguridad humana, a diferencia de los demás debates sobre seguridad en el ámbito de las Relaciones Internacionales, ha sido desarrollado en el nivel institucional (Naciones Unidas) y académico y, por ello, articula una agenda amplia (Derechos Humanos, Derecho Internacional Humanitario, Desarrollo). Asimismo, ha sido adoptado en los discursos y en la política exterior de los Estados, sobre todo Japón, Canadá y Noruega (BUZAN; HANSEN, 2009, p. 205).

Para comprender el surgimiento del concepto de seguridad humana en el seno de Naciones Unidas, es imprescindible recurrir al Informe sobre Desarrollo Humano del Programa de las Naciones Unidas para el Desarrollo (PNUD) de 1994․ Es este el primer documento, en el seno de las Naciones Unidas, que presenta el concepto de seguridad humana y lo relaciona al desarrollo humano.

Este Informe deja claro que no habrá paz a menos que los seres humanos disfruten de seguridad en su vida cotidiana. El fin de la guerra fría fue un marco importante en las relaciones internacionales, pues puso de manifiesto crecientes disparidades económicas entre los países y el surgimiento de una serie de conflictos armados con características distintas a los conflictos del periodo anterior (guerra fría).

Los conflictos asumieron carácter interno y sus causas no eran las tradicionales, es decir, territoriales, sino que ocurrían debido a factores étnicos o religiosos. Los ejemplos más emblemáticos fueron los conflictos en el territorio de la antigua Yugoslavia y en Ruanda, que llamaron la atención de la comunidad internacional y obligaron el Consejo de Seguridad de Naciones Unidas a aprobar operaciones de manutención de la paz con características distintas de las anteriores 9 . Se trató de intervenciones humanitarias, cuyos objetivos fueron proteger a la población civil y garantizar el respeto al derecho internacional humanitario ${ }^{10}$.

\footnotetext{
${ }^{8}$ PROGRAMA DE LAS NACIONES UNIDAS PARA EL DESARROLLO (PNUD). Informe sobre Desarrollo Humano 1994. México: Fondo de Cultura Económica, S.A. de C.V., 1994

${ }^{9}$ La resolución 827 (25 de mayo de 1993) del Consejo de Seguridad crea el Tribunal Penal Internacional para la antigua Yugoslavia. Por primera vez, el Consejo, invocando el Capítulo VII de la Carta de Naciones Unidas, declara que las violaciones graves de derecho internacional humanitario en el territorio de la antigua Yugoslavia constituían amenazas a la paz y a la seguridad (UNITED NATIONS SECURIY COUNCIL. Resolution 827 (1993). Doc. S/RES/827 (1993), 25 de mayo de 1993). En 1994, por los mismos motivos, el Consejo de Seguridad establece el Tribunal Penal Internacional para Ruanda (resolución 955 de 8 de noviembre de 1994) (UNITED NATIONS SECURITY COUNCIL. Resolution 955 (1994). Doc. S/RES/955 (1994), 8 de noviembre de 1994).

${ }^{10}$ Así como las resoluciones 827 (1993) y 955 (1994), la resolución 1970 (2011) respecto a la situación en Libia habla de las graves violaciones de derecho internacional humanitario que estaban ocurriendo contra la población del país. Sin embargo, las resoluciones sobre el conflicto en la antigua Yugoslavia y el conflicto en Ruanda afirman que las violaciones del derecho internacional humanitario constituían amenazas a la paz y la seguridad internacionales (Capítulo VII de la Carta de Naciones Unidas). La resolución 1970 (2011) condenó el uso de la fuerza por el gobierno libio en contra de la población civil, así como las graves violaciones de derechos humanos y del derecho internacional humanitario cometidas por los más altos funcionarios estatales, y consideró que el gobierno de Libia no estaba cumpliendo con su responsabilidad de proteger a su población. De ese modo, la resolución
} 
De ese modo, partir de 1990 se diversificó el uso de la "etiqueta humanitaria" en intervenciones de carácter militar. En ese nuevo contexto que se enmarca la paz y la seguridad el Consejo de Seguridad tiene un papel importante en los asuntos humanitarios, por medio de la ampliación del significado de 'la amenaza a la paz y la seguridad internacionales' a las 'crisis humanitarias'11.

Esta nueva percepción se encuentra plasmada en la 'Agenda para la Paz', de 1992, del ex secretario general de NNUU, Boutros Boutros-Ghali, que ha sido un referente para la comprensión del teatro de operaciones tras la guerra fría y del rol de $\mathrm{NNUU}^{12}$. Con el fin de la bipolaridad en las relaciones internacionales, el ex secretario presentó una revisión de la definición de seguridad $^{13}$, que relaciona en términos de pobreza, desigualdad, crecimiento poblacional. Es la tendencia a la 'securitización' de la ayuda o el sentimiento de que los desplazamientos poblacionales y las violaciones masivas de los Derechos Humanos son considerados como una amenaza para la seguridad internacional. Obsérvese cómo las víctimas pasan a ser introducidas en el discurso de la seguridad colectiva y del desarrollo global. Más allá de su condición de víctimas en situación de vulnerabilidad, una vez forzadas a moverse, se empieza a tener en cuenta la inseguridad humana en el proceso de desplazamiento. Al mismo tiempo se observa una política cada vez más restrictiva y vinculada en la gestión de los flujos migratorios, que tiende a criminalizar al migrante.

El Informe del PNUD (1994) pretendía demostrar que muchos de los conflictos del pos guerra fría ocurrían por crecientes disparidades y privaciones socioeconómicas y, de ese modo, el desarrollo integral sería fundamental para que se alcanzase la seguridad humana. Desarrollo humano y seguridad humana son dos conceptos interdependientes y el citado Informe presenta un nuevo paradigma de desarrollo para enfrentar los desafíos que supone la seguridad humana. Este paradigma pone el ser humano en el centro del desarrollo y contempla la sostenibilidad desde una perspectiva integral (pensar en el bienestar de las generaciones futuras) (PNUD, 1994, p. 5).

1970, además condenar las violaciones, toma otras medidas, como referir la situación al Tribunal Penal Internacional (UNITED NATIONS SECURITY COUNCIL. Resolution 1970 (2011). Doc. A/ RES/1970 (2011), 26 de febrero de 2011).

${ }^{11}$ BOUCHET-SAULNIER, Françoise, Dictionnaire pratique du Droit Humanitaire, Editions La Découverte \& Syros, Paris, 1998, p.241.

12 "analysis and recommendations on ways of strengthening and making more efficient within the framework and provisions of the Charter the capacity of the United Nations for preventive diplomacy, for peacemaking and for peace-keeping". Vease: UN GENERAL ASSEMBLY, “An Agenda for Peace: Preventive diplomacy and related matters", A/RES/47/120, de 18 dicember 1992

13 "Emphasizing that international peace and security must be seen in an integrated manner and that the efforts of the Organization to build peace, justice, stability and security must encompass not only military matters, but also, through its various organs within their respective areas of competence, relevant political, economic, social, humanitarian, environmental and developmental aspects". (A/ $\mathrm{RES} / 47 / 120.4^{\circ}$ parr.) 
Desde un nuevo paradigma de desarrollo humano, el Informe invita a los Estados a pensar en un concepto de seguridad humana más allá del concepto tradicional que predominó en la guerra fría, de protección de las fronteras nacionales contra amenazas externas (PNUD, 1994, p. 3). Se afirma que, después de la guerra fría, los conflictos tienen carácter interno y las personas están más preocupadas con otros tipos de amenazas, como el hambre, la miseria, las enfermedades, el desempleo etc. (PNUD, 1994, p. 25).

La definición de seguridad humana presentada en el Informe del PNUD posee dos aspectos relevantes en el presente estudio: 1. Significa seguridad contra las amenazas crónicas (hambre, enfermedades etc.) y; 2. "Protección contra alteraciones súbitas y dolorosas de la vida cotidiana". De ese modo, la seguridad humana presenta dos componentes: libertad respecto del miedo y libertad respecto de la necesidad (PNUD, 1994, pp. 26-27).

El Informe también presenta las dimensiones de la seguridad humana: seguridad económica, seguridad alimentaria, seguridad en materia de salud, seguridad ambiental, seguridad personal, seguridad de la comunidad, seguridad política (PNUD, 1994, p. 28). Además, la seguridad humana presenta un aspecto global, pues existen amenazas que son transnacionales y afectan a toda la comunidad internacional, como por ejemplo la degradación del medio ambiente, las migraciones, el tráfico de estupefacientes, el terrorismo etc. (PNUD, 1994, pp. 38-39).

Ya en 2003, la Comisión sobre Seguridad Humana divulgó el Informe Human Security Now que presenta las ideas clave del Informe del PNUD (1994): seguridad respecto del miedo y seguridad respecto de la miseria. El Informe de 2003 añade que la seguridad humana significa la protección de las personas contra amenazas latentes y, también, su empoderamiento para que puedan conducir sus propias vidas. El Informe hace también una serie de recomendaciones, de largo y corto plazo, para alcanzar la protección y empoderamiento de las personas. Expresando con ello que:

\footnotetext{
"seguridad humana significa proteger las libertades fundamentales: libertades que constituyen la esencia de la vida. Significa proteger al ser humano contra las situaciones y las amenazas críticas (graves) y omnipresentes (generalizadas). Significa utilizar procesos que se basan en la fortaleza y las aspiraciones del ser humano" (Commission on Human Security, 2003, p.3).
}

Como se pone en evidencia, la seguridad humana vincula las diferentes libertades: libertad frente a las privaciones, libertad frente al miedo y libertad para actuar en nombre propio. Y demanda una voluntad y acción "por forjar una alianza mundial a fin de reforzar las políticas institucionales que vinculan a la persona y al Estado, y el Estado con el mundo. O sea que la seguridad humana agrupa los elementos de seguridad, de derechos, de desarrollo" (Commission on Human Security, 2003, p.2). 
El concepto de seguridad humana recibe otro impulso, en 2006, a partir de la formación del Grupo de Amigos de la Seguridad Humana (FSH) ${ }^{14}$, copresidido por Japón y México, e integrado por 34 países. Se trata de un foro informal entre los Estados miembros y organizaciones internacionales cuya finalidad es analizar el concepto y alcance de la seguridad humana desde diferentes ángulos "de tal manera que se llegue a una comprensión común sobre la seguridad humana y se exploren esfuerzos de colaboración para la integración del concepto en las actividades de Naciones Unidas"15.

En su Informe de $2005^{16}$, el Secretario-General de Naciones Unidas afirma que las amenazas a la paz y a la seguridad en el siglo XXI no son solo el terrorismo, los conflictos internacionales y las armas de destrucción en masa, sino que las enfermedades mortales, la pobreza o la degradación ambiental pueden provocar, también, consecuencias catastróficas (párrafo 78).

El Secretario-General expresa que, ante amenazas latentes, es decir, las amenazas a la paz y a la seguridad, el Consejo de Seguridad está autorizado a utilizar la fuerza, incluso de forma preventiva. Sin embargo, afirma el Secretario que el genocidio, la limpieza étnica y otros crímenes de lesa humanidad también serían considerados amenazas a la paz y la seguridad internacionales. De ese modo, el Secretario General pone de manifiesto que la responsabilidad de los Estados, en primer lugar, es la de proteger a sus poblaciones ${ }^{17}$. No obstante, si las autoridades nacionales no pueden o no están dispuestas a proteger a su población contra estas graves amenazas, cabe a la comunidad internacional la responsabilidad de utilizar todos los medios disponibles para proteger a las personas. En último caso, el Consejo de Seguridad deberá tomar las medidas necesarias de acuerdo con la Carta de Naciones Unidas (párrafos 125 y 135).

En 2010, el Secretario-General presenta un Informe sobre Seguridad Humana ${ }^{18}$. Afirma que la definición de seguridad humana surge como respuesta a las amenazas actuales, que son múltiples, complejas, interrelacionadas y

\footnotetext{
${ }^{14}$ Según las iniciativas de FSH, los progresos han sido positivos y han corroborado para la materialización e integración del concepto de seguridad humana dentro de la comunidad internacional que se observa a través del reposicionamiento de los Estados ante distintas amenazas contra la seguridad humana.'Friends of Human Security" Meeting, 19 October 2006, New York. Disponible en: http://www.mofa.go.jp/policy/human_secu/friends/summary0610.html.

15 Disponible en: https://www.iidh.ed.cr/multic/default_12.aspx?contenidoid=8c1a302f-f00e4f67-b3e6-8a3979cf15cd\&Portal=IIDHSeguridad.

${ }^{16}$ NACIONES UNIDAS. Un concepto más amplio de la libertad: desarrollo, seguridad y derechos humanos para todos. Informe del Secretario General. A/59/2005, 21 de marzo de 2005.

${ }_{17}$ R2P que será abordado a posterior

18 NACIONES UNIDAS. Seguridad humana. Informe del Secretario General. Doc. A/64/701, 8 de marzo de 2010. Aunque el informe no trate directamente el tema de la Trata de mujeres, el mismo indirectamente afirma que la ONU viene tomando medidas para proteger los grupos mas vulnerables en situaciones de conflictos (pár. 51). En este sentido, Kofi Anan definió como amenazas a la seguridad humana las violaciones masivas de derechos humanos, el desplazamiento masivo de poblaciones, el terrorismo internacional, la pandemia del SIDA, el tráfico de armas, drogas, personas y las catástrofes medioambientales (Doc. ONU A/55/1, de 30 de agosto de 2000, pár. 31).
} 
transnacionales. Además, el concepto de seguridad humana respeta la protección y el empoderamiento de las personas y significa una vida libre del miedo y de la miseria. Ello no presupondría el uso de la fuerza contra la soberanía de los Estados (párrafo 19).

En este sentido, el Secretario-General refuerza que los Estados deben hacer frente a las amenazas a la seguridad humana de sus poblaciones. Reitera que la responsabilidad de proteger es un concepto relacionado a la protección de las personas contra los crímenes de lesa humanidad y los crímenes de guerra y la comunidad internacional debe intentar evitar su ocurrencia, pues causan crisis humanitarias y grandes pérdidas de vidas humanas (párrafo 124).

En el Informe de 2010, el Secretario-General presenta las principales amenazas a la seguridad humana: la crisis financiera mundial, la inestabilidad de los precios de los alimentos y la inseguridad alimentaria, la propagación de enfermedades infecciosas, el cambio climático. Además, presenta la necesidad de prevenir conflictos violentos y del mantenimiento y consolidación de la paz. Pues las amenazas inciden en mayor situación de vulnerabilidad en las mujeres, niños/as y desplazados internos (párrafo 51).

\subsection{Críticas al concepto de seguridad humana: PNUD}

Como puntualizado anteriormente, el Informe de 1994 del PNUD fue el primer documento de Naciones Unidas que trató del concepto de seguridad humana y contribuyó para poner el ser humano como el referente de la seguridad, posición siempre ocupada por los Estados (McFARLANE; KHONG, 2006, p. 227). Sin embargo, el Informe del PNUD fue criticado por presentar un concepto muy amplio de seguridad humana, que engloba distintas amenazas y, además, no establece cuáles son las amenazas a la seguridad del individuo, si son los conflictos (libertad respecto del miedo) o el hambre, las enfermedades etc. (libertad respecto de la miseria).

McFarlane y Khong (2006, pp. 237-238) afirman que este concepto posee una doble orientación, es vertical, pues incluye desde las organizaciones internacionales hasta los individuos, y horizontal, incluyendo cuestiones económicas, ambientales, sociales, de salud, etc. Para los autores, el abordaje horizontal lleva a falsas esperanzas y prioridades, a la vez que, si la seguridad humana lo incluye todo, ¿cuál debe ser la prioridad? De ese modo, hay confusión causal, es decir, las amenazas son diversificadas, lo que hace que sea una tarea difícil determinar sus causas.

En el seno de Naciones Unidas, además del Informe del PNUD de 1994 y de los Informes del Secretario-General, otros órganos y agencias, como UNICEF (United Nations Children's Fund) y UNHCHR (United Nations High Commissioner for Human Rights), no han utilizado el término "seguridad 
humana" en sus documentos. Tampoco el Consejo de Seguridad lo ha utilizado en sus resoluciones, sobre todo porque determinadas potencias (Estados Unidos, China y Rusia) conservan su foco estatocéntrico en las cuestiones de amenaza a la paz y la seguridad internacionales. Sin embargo, Japón, Canadá y Noruega utilizan el término "seguridad humana" en sus programas de ayuda al desarrollo, que forman parte de sus políticas exteriores (McFARLANE; KHONG, 2006, p. 10).

No se puede obviar que el Informe del PNUD se convirtió en un importante documento, pues sirvió de base para futuros desarrollos respecto al concepto de seguridad humana. McFarlane y Khong (2006, pp. 149-150) afirman que hay, en el Informe, una contestación implícita de la soberanía de los Estados y se sugiere la reforma del Consejo de Seguridad. Por esta razón, el documento no fue bien recibido por el órgano.

Sin embargo, las resoluciones del Consejo de Seguridad en el periodo del pos guerra fría contemplaron la protección de las personas en los conflictos armados (casos de Bosnia ${ }^{19}$, Ruanda ${ }^{20}$ y Libia ${ }^{21}$ ). Ello significó un marco en las resoluciones del órgano, que privilegiaron la seguridad del Estado, y no del individuo. En los Informes del Secretario-General (2005 y 2010), queda claro que cabe a los Estados la responsabilidad principal por la seguridad de su población, es decir, por la seguridad humana.

McFarlane y Khong (2006) han puesto de manifiesto que tanto las amenazas a la seguridad física de las personas, como los conflictos armados que causan graves problemas (refugiados, desplazados internos, hambre etc.) y que son considerados crímenes de lesa humanidad y crímenes de guerra, movilizan la atención de la comunidad internacional y del Consejo de Seguridad, mientras que las demás amenazas (horizontales) no llevan a que el órgano actúe rápidamente, es decir, no se configuran en situaciones de amenaza a la paz y la seguridad internacionales. Contrariando así la finalidad expresa de la seguridad humana que es salvaguardar a dignidad y las libertades (PNUD, 1994, parágrafo. 143). De acuerdo con Peral (2005, p. 16), el problema está en cómo dar contenido práctico al concepto.

\subsection{Responsabilidad de proteger en el contexto de la seguridad humana}

Como vimos anteriormente, la noción de Seguridad humana esta estructurada como un medio para liberar a las personas tanto de las incertidumbres y temores, como también de las necesidades materiales básicas para la subsistencia y vida digna (ICISS, 2001). De modo a conferir contenido práctico al programa

\footnotetext{
19 S/RES/836/1993, de 04 de junio de 1993.

20 S/RES/955/1994, de 08 de noviembre de 1994.

${ }^{21} \mathrm{~S} / \mathrm{RES} / 1970 / 2011$, de 26 de febrero de 2011.
} 
en el seno de Naciones Unidas, la Comisión de Seguridad Humana elaboró el informe "Seguridad Humana, ahora" (2003), en que había analizado los ámbitos relacionados a pobreza y conflictos y evidencia contextos en que la seguridad humana está amenazada. Las principales amenazas señaladas a la seguridad son aquellas que generan situaciones de vulnerabilidades: económicas y sociales, la integración social (en este contexto los movimientos migratorios), v. de la seguridad internacional tradicional (conflictos y amenazas de carácter transnacional) y v. de la seguridad interna (violencia social), v. institucionalizada y delincuencia (UNESCO, 2005, pp. 101-180).

El principio de la Responsabilidad de Proteger (R2P) está directamente relacionada a la de Seguridad Humana, será aquí entendido de modo complementario (a la Seguridad humana), subsidiario (respecto a la acción del Estado) y multilateral (en cuanto a su ejercicio) (PERAL, 2005, p.16). Esta percepción queda evidenciada a través del "informe sobre la responsabilidad de proteger", elaborado por la Comisión Internacional sobre Intervención y Soberanía del Estado (ICISS) ${ }^{22}$ que particulariza que la R2P a las personas corresponde al Estado, pero que de forma subsidiaria se posibilita la intervención humanitaria.

En ese sentido, desde la perspectiva de la doctrina de la R2P, sobre todo de la forma que la ha aplicado el Consejo de Seguridad, la seguridad humana está relacionada a la protección del individuo en situaciones de conflictos armados. El Informe de la ICISS presenta los principios básicos de la Responsabilidad de proteger: 1) la responsabilidad primera de la protección de los individuos es de los Estados y 2) en situaciones de conflictos armados, si el Estado no está dispuesto o no es capaz de asegurar la protección de las personas, el principio de no intervención da lugar a la responsabilidad de proteger de la comunidad internacional. La obligación recae, así, en el Consejo de Seguridad, el órgano de Naciones Unidas responsable por el mantenimiento de la paz y la seguridad internacional (ICISS, 2001).

El Informe presenta tres tipos de responsabilidades inherentes a la doctrina de la Responsabilidad de proteger: a) la responsabilidad de prevenir; b) la responsabilidad de reaccionar (aquí caben las medidas necesarias para responder a las situaciones de graves violaciones de Derechos Humanos, como medidas de coerción o, incluso, intervención armada); c) la responsabilidad de reconstruir (después de una intervención, prestar la asistencia a la reconstrucción). Un aspecto importante que pone de manifiesto el Informe es el carácter excepcional y extraordinario de las intervenciones humanitarias, es decir, mediante la doctrina de la responsabilidad de proteger y, así, para que

${ }^{22}$ INTERNATIONAL COMMISSION ON INTERVENTION AND STATE SOVEREIGNTY (ICISS). The Responsibility to Protect. Canadá: International Development Research Centre, Diciembre de 2001. 
tengan lugar las intervenciones, deben ocurrir graves y sistemáticas violaciones de Derechos Humanos, como el genocidio, la limpieza étnica u otros crímenes de lesa humanidad (ICISS, 2001).

\title{
Peral destaca:
}

\begin{abstract}
"Dos cuestiones, la primera más teórica y la segunda de índole práctica, son prioritarias. Por un lado, es preciso unificar las ideas de responsabilidad colectiva internacional y de garantía a todas las personas, comenzando por las poblaciones en peligro, de un marco de seguridad que les permita el disfrute efectivo de sus derechos humanos. Así, la responsabilidad de proteger sería el modo en que la comunidad internacional garantiza la seguridad humana en las situaciones extremas en que el Estado, responsable primario frente a su propia población, no puede o no quiere hacerlo. Por otro lado, la disposición a garantizar subsidiariamente la seguridad humana ha de traducirse en el drástico aumento de las capacidades civiles internacionales, tanto de las NU y las Org. Regionales, como en el ámbito de la acción exterior de los Estados. (...) Cuando se trata de injerencia para salvar a poblaciones en peligro, la clave no reside tanto en la justificación de los fines proclamados cuanto en el recurso a los medios adecuados y en la proporción adecuada para atender los fines proclamados. Si el concepto de seguridad humana logra poner el énfasis en la legitimidad de los medios de acción, todo esfuerzo habrá merecido la pena (2005, p.16).
\end{abstract}

En el documento de la World Summit Outcome $e^{23}$, de 2005, los párrafos $138^{24}$ y $139^{25}$ ponen de manifiesto la doctrina de la responsabilidad de proteger las poblaciones del genocidio, crímenes de guerra, limpieza étnica y crímenes de lesa humanidad, reforzando los principios del Relatorio de la Comisión Internacional sobre Intervención y Soberanía del Estado. Como hemos

${ }^{23}$ UNITED NATIONS. 2005 World Summit Outcome. A/60/L.1, 15 de septiembre de 2005.

24 "Each individual State has the responsibility to protect its populations from genocide, war crimes, ethnic cleansing and crimes against humanity. This responsibility entails the prevention of such crimes, including their incitement, through appropriate and necessary means. We accept that responsibility and will act in accordance with it. The international community should, as appropriate, encourage and help States to exercise this responsibility and support the United Nations in establishing an early warning capability" (UNITED NATIONS, 2005).

25 "The international community, through the United Nations, also has the responsibility to use appropriate diplomatic, humanitarian and other peaceful means, in accordance with Chapters VI and VIII of the Charter, to help protect populations from genocide, war crimes, ethnic cleansing and crimes against humanity. In this context, we are prepared to take collective action, in a timely and decisive manner, through the Security Council, in accordance with the Charter, including Chapter VII, on a case-by-case basis and in cooperation with relevant regional organizations as appropriate, should peaceful means be inadequate and national authorities manifestly fail to protect their populations from genocide, war crimes, ethnic cleansing and crimes against humanity. We stress the need for the General Assembly to continue consideration of the responsibility to protect populations from genocide, war crimes, ethnic cleansing and crimes against humanity and its implications, bearing in mind the principles of the Charter and international law. We also intend to commit ourselves, as necessary and appropriate, to helping States build capacity to protect their populations from genocide, war crimes, ethnic cleansing and crimes against humanity and to assisting those which are under stress before crises and conflicts break out" (UNITED NATIONS, 2005). 
destacado, el Informe de 2005 sobre Seguridad Humana del Secretario General reitera los párrafos 138 y 139 del documento de la Cumbre. En relación a la Trata de Personas, no entra directamente en la materia, apenas señala la necesidad de poner fin al abuso y la explotación sexual del personal de NNUU (párrafo 97).

De acuerdo con Peral (2005, p.3) y NNUU (Doc. A760/L.1, 2005, párr. 8), el concepto de la R2P será un concepto vacío de contenido si no se traduce de modo urgente en la creación de medio civiles destinados a la protección efectiva de los derechos básicos en caso de amenaza a la seguridad humana.

\section{Como contribuye la Seguridad Humana en el enfrentamiento a la Trata de seres humanos}

\section{1 ¿Cómo está estructurada la política anti-trata?}

Si volvemos a la expansión del concepto de seguridad propuesto por D. Baldwin (1997, p.6): ¿Seguridad para quién? ¿Para qué?”, podemos observar algunas particularidades en el Enfrentamiento a la Trata de Personas (ETP).

La primera en el marco de la lucha contra el crimen transnacional organizado, puesto que la política internacional anti Trata de personas rebota a la agenda internacional a partir de la firma del Protocolo para Prevenir, Reprimir y Sancionar la Trata de Personas, especialmente Mujeres y niños, que complementa la Convención de las Naciones Unidas contra la Delincuencia Organizada Transnacional para Prevenir de Palermo en el año 2000 (Protocolo de Palermo) ${ }^{26}$.

La segunda es referente al enfoque estatocéntrico sobre la seguridad. Es decir, una visión tradicional donde el Estado es el principal objeto de seguridad $^{27}$. Luego prima el control de fronteras (como forma de garantizar su seguridad ante amenazas externas), cuestiones de lucha contra el crimen transnacional organizado, tocando tangencialmente el enfoque de los Derechos Humanos. No es un enfoque aislado, puesto que el mismo tiene su base en el propio referente internacional -Protocolo de Palermo-, que estructura el ETP desde tres pilares, también conocidos como las 3 'P's: Prevención, Persecución y Protección $\left(\right.$ Art.2) ${ }^{28}$. El actual ETP prima por el combate al crimen

\footnotetext{
${ }^{26}$ Resolución 55/25 de la Asamblea General de Naciones Unidas, de 15 de noviembre de 2000.

${ }^{27}$ Queda evidenciado desde el marco internacional en la lucha contra la Trata, que es la Convención contra el Crimen Transnacional Organizado (2000) y un de sus protocolos adicionales, el Protocolo para Prevenir Reprimir y Sancionar la Trata de Personas, especialmente mujeres y niños, adicional a la Convención.

${ }^{28}$ Como dispone el artículo 2 del Protocolo de Palermo: "The purposes of this Protocol are: (a) To prevent and combat trafficking in persons, paying particular attention to women and children; (b) To protect and assist the victims of such trafficking, with full respect for their human rights; and (c) To promote cooperation among States Parties in order to meet those objectives".
} 
transnacional organizado, a través de persecución al crimen y sanción de los tratantes. De ahí, que el actor estatal asume el protagonismo en lo referente a la secutiritización $y$, por otro lado, delega la protección y asistencia a la sociedad civil. Y la protección queda subrealizada mediante acciones puntuales de sensibilización o vía prevención de la 'inmigración irregular' que muchas veces ocurre mediante la gestión de los flujos migratorios.

La tercera particularidad que observamos es la que articula la seguridad del Estado como principal preocupación en el ETP. En este contexto, la lucha contra la amenaza de la inmigración representa una prioridad en materia de seguridad (IONESCU, 2007, 218). Esta respuesta estatal se forja en política de justicia criminal que se centra en la prohibición de libertad de movimiento (penalización) y el control (ejecución) que han generado leyes de inmigración más estrictas que, en muchos casos, han obligado a utilizar las vías irregulares para el cruce de fronteras, o, como vulgarmente se conoce, "entrar por la puerta trasera". Este acercamiento miope ${ }^{29}$, de combate al crimen transnacional, reduce la Trata a uno de sus enfoques, el control de la movilidad internacional, ignorando la causa principal de su existencia y las consecuencias del proceso violatorio de derechos humanos y la perpetuación de un contexto de inseguridad humana.

Como se ha evidenciado, existe una polifonía en el ETP. Uno de ellos es su comprensión como una violación masiva de Derechos Humanos, una forma contemporánea de esclavitud (ocurre mediante el dominio, sumisión, restricción de libertad y la explotación del trabajo en condiciones denigrantes (CORRÊA DA SILVA, 2011), una violencia de género (también está relacionado con una discriminación contra la mujer, como consecuencia de un sistema desigual y excluyente que se facilita la causa y consecuencia de la Trata de mujeres), es también un crimen contra la humanidad (art. 7 del Estatuto de Roma de la CPI) $\mathrm{y}$, que consiste en alejar una persona de su entorno, u origen, con la finalidad de explotarla en otro lugar de destino. Otro enfoque conferido a la Trata está relacionado a la inmigración irregular y al combate al crimen transnacional organizado. "Ante la abundancia terminológica, se halla una confusión conceptual que franquea el debate sobre la Trata de seres humanos, generando impactos negativos tanto para la protección y asistencia integral de las víctimas cuanto para la prevención y el auto-reconocimiento en cuanto víctimas de violación de derechos humanos" (CORREA DA SILVA, 2014, p.225).

Está claro que estamos delante de un fenómeno complejo y polifacético, y para su comprensión es necesario no solo comprender su concepto, como también interpretar su configuración desde una perspectiva multidisciplinar,

29 "El tratamiento de la trata de como delito significa que tanto los tratantes como las víctimas están recibiendo el mismo tratamiento de persecución y sanción (expulsión y privación de libertad). Esto es una consecuencia directa de las tomas de decisiones ignorando las consideraciones humanas en la formulación de políticas de enfrentamiento a la Trata” (IONESCU, 2007, p.218).

Araucaria. Revista Iberoamericana de Filosofía, Política y Humanidades, año 18, n 36. Segundo semestre de 2016. Pp. 217-240. ISSN 1575-6823 e-ISSN 2340-2199 doi: 10.12795/araucaria.2016.i36.10 
que va más allá de un problema migratorio, o una actividad criminal que conlleva a la práctica de un delito y violación de derechos. Asimismo, no se puede obviar que es una práctica violatoria de derechos que está legitimada por la falta de consciencia, las conductas socioculturales y las transacciones económicas lucrativa-explotación. Para un enfrentamiento integral al problema es necesario tanto factores sistémicos como acciones que ataquen a sus causas que están relacionadas con la pobreza, la discriminación contra las mujeres y la capacidad política e institucional para proteger a las víctimas y perseguir a los tratantes ${ }^{30}$. De ahí que el marco conceptual de la seguridad humana proporciona el cuadro necesario para estudiar las causas estructurales de la Trata de personas mientras que destaca eficaces, innovadores y soluciones sostenibles para la lucha contra esta forma contemporánea de esclavitud.

\subsection{Del concepto de Trata y su conexión con la seguridad humana: La estructura de explotación que genera la inseguridad}

Para el concepto de Trata de Personas, utilizamos el referente internacional sobre la materia: el Protocolo de Palermo (Art. $\left.3^{\circ}\right)^{31}$, que ofrece una definición de Trata de Personas que contiene tres elementos constitutivos: una acción; los medios; y la finalidad de explotación. Estos elementos están interrelacionados, ya que se trata de una acción que se asienta en la captación, en el transporte, en la acogida de personas, determinando una conducta delictiva con la finalidad de explotación. Para obtener el consentimiento de una persona que tenga autoridad sobre otra, se utiliza amenaza, la fuerza u otras formas de coacción: el rapto, el fraude y el engaño, el abuso de poder frente a una situación de vulnerabilidad, así como la concesión o la recepción de pagos o beneficios; su fin es la explotación.

Entendemos que coexisten diferentes formas de Trata de personas, ya que la explotación del trabajo - constriñendo la víctima a realizar trabajos forzados, sin remuneración o con aislamiento, de servidumbre por deudas (supuestamente contraídas, para fines sexuales comerciales, sometiendo a la persona a prostitución o a la explotación sexual). En estos términos, defendemos que sobreviene explotación cuando hay: insuficiencia de garantía de derechos laborales, jornada de trabajo que expone a la persona a condiciones inhumanas, degradantes y violatoria de Derechos Humanos consagrados por el orden internacional. Otras formas de explotación aunque subnotificadas,

${ }^{30}$ En este sentido: http://www.weinstitute.org/human-trafficking.html

31 "(a) "Trafficking in persons" shall mean the recruitment, transportation, transfer, harbouring or receipt of persons, by means of the threat or use of force or other forms of coercion, of abduction, of fraud, of deception, of the abuse of power or of a position of vulnerability or of the giving or receiving of payments or benefits to achieve the consent of a person having control over another person, for the purpose of exploitation. Exploitation shall include, at a minimum, the exploitation of the prostitution of others or other forms of sexual exploitation, forced labour or services, slavery or practices similar to slavery, servitude or the removal of organs;" (Art. 3 a. Protocolo de Palermo). 
son las modalidades de: remoción de órganos y tejidos humanos, para fines de adopción irregular, para explotación del trabajo infantil y uso del vientre de alquiler. El uso de la terminología de Trata a diferentes procesos (en algunos casos paradójico) pueden conducir a una variedad de resultados, que generan el "mínimo común” de la explotación (DAVIDSON, 2008, p.11).

La definición de Trata no describe solo un acto unitario que lleva a un resultado específico, sino que se refiere a un proceso (captación, transporte y control $)^{32}$ que se puede organizar en una variedad de formas e involucrar una gama de diferentes acciones y resultados (DAVIDSON, 2008, p.11). En definitiva, permite comprender que se inicia con un acto particular del tratante, que utiliza de medios capciosos y se aprovecha de una situación de vulnerabilidad generada por un contexto de inseguridad humana. A partir de estas dos acciones iniciales se configura la explotación. Con este fin, se cosifica al ser humano y se vulnera su derecho a no ser sometido a la esclavitud, al trato inhumano y denigrante. Lo que corrobora la situación de amenaza a la seguridad humana y genera la vulnerabilidad de las personas. Este enfoque contribuye a:

Juridical approaches to trafficking, however, do not perceive anything amiss prior to the commission of an exploitative act. Yet, it is untenable to assume that human trafficking only begins with a malicious action or ends when traffickers are arrested and successfully prosecuted. Additionally, being a product of public law, the AMP model inherently retains the primacy of states as leading anti-human trafficking initiatives (KREIDENWEIS y HUDSON, 2015, p.69).

Una de las consecuencias del enfoque estatocéntrico, en el ETP, es ignorar la importancia del componente "agencia" del proceso de la Trata. Un ejemplo claro son las medidas de protección que están concertadas en la necesidad de "rescate" de las víctimas, que las mismas colaboren para la sanción de los tratantes o la devolución a las mismas condiciones socioeconómicas que le empujaron hacia la Trata (IONESCU, 2005, p. 219). Existe una carencia de soluciones que pasen por la capacitación de las personas en situación de vulnerabilidad, poniendo a su disposición servicios y apoyo socioeconómico, incluso si se les ofrezca una alternativa socioeconómica que le retire de la inseguridad humana.

\footnotetext{
${ }^{32}$ Así mismo: "It is imperative we understand that trafficking often implies the consent of the victim, who, with no other options, agrees to the terms put forward by the traffickers. In both sexual and labor exploitation cases, the number of victims that have been kidnapped, deceived and/or forced to work is considerably smaller than that of the people who knowingly agree to being trafficked. Actually many victims do not perceive themselves as such, since they receive money for their work and since exploitation in a rich country is preferable to the chronic poverty back home. Therefore, it is no surprise they are reluctant to reporting their situation as it inevitably leads to their deportation. (IONESCU, 2005, p. 219).
} 
Otro factor que no se puede evadir en el actual ETP es que casi ignora la dimensión humana de la Trata. Es decir, la persona víctima de Trata, como también ignora la demanda existente en los países de origen.

\section{3 ¿Cómo se conecta con el tema de la seguridad?}

En el acercamiento de la Trata de personas a la seguridad humana no se puede eludir que la decisión de migrar ocurre en un contexto de movilidad de inseguridad humana. Existe una relación directa entre el factor de empuje, o causante de la migración, como ejemplo de las amenazas a la seguridad humana $^{33}$, las posibilidades de realizar legalmente el mismo y las diferentes visiones de migraciones de los Estados.

Naciones Unidas identificó seis principales amenazas emergentes a la seguridad humana: el crecimiento incontrolado de la población, las disparidades en las oportunidades económicas, la migración internacional excesiva, la degradación ambiental, la producción y el tráfico de drogas y el terrorismo internacional (UNESCO, 2005, p. 103). Estas amenazas trascienden las fronteras nacionales, constituyen un desafío para la seguridad humana. La Trata de personas está en la intersección de todas las amenazas identificadas anteriormente, ya que las amenazas son los factores de empuje y crean un terreno fértil para aumentar algunas de estas amenazas (PATI, 2014, p. 32).

Los condicionantes que generan diferentes situaciones de vulnerabilidad y que les impide tener una vida digna en los países de origen, a la vez encuentra trabas con las trabas a la libertad de movimiento en los países de destino. Lo que significa afirmar que la migración es un proceso que refleja el mundo desigual y de las facilidades de movilidad que algunos poseen por su país de origen, como ejemplo de los originarios de los países que conforman la OCDE (UE), con altos ingresos que cuentan con facilidades para el cruce de fronteras en oposición a aquellos provenientes de países empobrecidos, de países en conflictos o, países marcados por regímenes autocráticos que, debido a la instabilidad política, económica y fuerte represión y uso constante de violencia, compromete directamente la seguridad humana.

En el actual escenario internacional, convulso, no se observan cada uno de los factores condicionantes de la Trata; es decir, no se observa cada flujo individualmente ni se clasifica según su factor de empuje y lo clasifica según el factor de empuje. Desde la aproximación a la seguridad humana se considera “insostenible suponer que el tráfico de personas sólo comienza con una acción maliciosa o termina cuando los traficantes están detenidos y procesados con éxito" (KREIDENWEIS y HUDSON, 2015, p. 69).

${ }^{33}$ Naciones Unidas orienta que las amenazas originadas de las vulnerabilidades pueden ser: económicas y sociales; de integración social; político-institucionales; de la seguridad internacional (como los conflictos y amenazas de carácter transnacional); de seguridad interna (violencia social y delincuencia, violencia institucionalizada, violencia simbólica) (UNESCO, 2005, p.103-180). 
La ampliación del ETP en conexión con la seguridad humana se acerca a la seguridad de una manera analítica ventajosa, ya que existe la posibilidad de un cambio de referente ('del estado para los individuos') y de enfoque (de las amenazas militares al estado de las amenazas políticas, económicas, ambientales y socioculturales). Esta disposición puede ser particularmente beneficiosa a las víctimas, cuando se utiliza como un vehículo para ayudar a reunir recursos y atención por parte de los responsables políticos para problemas de seguridad no convencionales (KREIDENWEIS y HUDSON, 2015, p. 70).

Se sugiere que el uso del enfoque de la Seguridad Humana para el ETP sería un importante catalizador de los diferentes esfuerzos, actores y agencias para enfrentar la Trata a nivel global y local (KREIDENWEIS y HUDSON, 2015, p. 72). Además, la perspectiva de Seguridad Humana ha sido capaz de interconectar los diferentes componentes de la seguridad y ha dado lugar a un equilibrio entre el Estado y la noción de seguridad centrada en las personas (FRERKS, 2008, p.12).

\section{Consideraciones finales: Seguridad humana como una herramienta descriptiva y prescriptiva en términos de política anti-Trata}

La definición de seguridad humana se caracteriza por cuatro componentes claves: es universal, interdependiente, preventiva y orientada a las personas. Para lograr su realización es imprescindible un enfoque integral (desde su aspecto multisectorial), contextualizado y preventivo, que sea capaz de identificar eficazmente las amenazas humanas existentes en el proceso de Trata (desde la comprensión de sus causas y consecuencias). De ahí la esencialidad de vincular sus tres enfoques: libertad del miedo (la actuación del crimen transnacional organizado y otros grupos delictivos como amenaza de la seguridad); libertad de la necesidad (situación de pobreza y no desarrollo); y, libertad para vivir con dignidad (discriminación, situación de exclusión y ofensas a los derechos humanos). En este escenario, el Estado juega un papel cardinal como garante de la seguridad (física, económica, social) y de los derechos y libertades fundamentales. No obstante, abandonamos la perspectiva estatocéntrica para una aproximación a los Derechos Humanos, en la cual se hace necesario reconocer y potenciar la participación activa de la sociedad civil organizada.

Dicho esto, se hace ineludible a la reorientación de la postura estatal en términos de seguridad, que ya no puede apenas limitarse a la protección de la integridad territorial y su soberanía, sino que debe incluir los elementos de la Seguridad Humana. No se trata de una postura neutral, puesto que implica la aplicación de políticas públicas, tanto para enfrentar las amenazas a la seguridad humana como para mejorar el bienestar y la seguridad de las 
personas y el respeto a su dignidad humana (TABUSCA, 2015, p. 259). El ETP, desde la aproximación a la Seguridad Humana, podría empujar a la eliminación de las principales causas que generan la vulnerabilidad de las personas, como la pobreza, la desigualdad y la exclusión social horizontal, la falta de representación política, la violencia (estructural y de género), la falta de prevención y sensibilización en temas de Trata (TABUSCA, 2015, p.264).

A partir de su concepto, su consecuencia al tejido social y demás implicaciones político-económicas y jurídicas, se percibe que la Trata genera la inseguridad humana al ser también una ofensa directa a los Derechos Humanos. Su naturaleza criminal añadida a sistemas legales insuficientes para la prevención y represión del crimen, sanción de los criminales y protección de las víctimas genera una complejidad para su erradicación. Es manifiesto que muchas de las situaciones de Trata, que son generadas por la inseguridad humana, son expresiones de un mercado global muy poco regulado, discriminatorio e ilegal. Donde personas que, ante la necesidad, buscan condiciones de vida digna y son atraídos por las oportunidades de trabajo, y se quedan atrapadas en los mecanismos de explotación de trabajo indecentes en la cadena de suministro global (TAVANTI, TUCKER, WANGERIN. 2015, p.10).

La adopción del enfoque de seguridad humana en el ETP además de ser un descriptor global de tráfico, puede ser un dispositivo eficaz para potencializar una acción política más amplia y oportuna, como las iniciativas de rehabilitación para las víctimas, y la investigación para identificar indicadores tempranos de la Trata de personas y los factores sociales que promueven la Trata enfocados. (KREIDENWEIS y HUDSON, 2015, p. 81). Lo que significa afirmar que la capacidad de los Estados de ejecutar una respuesta política integral en sus sistemas de justicia, las prácticas de aplicación de la ley, y la cooperación con las empresas y organizaciones de la sociedad civil están en el centro de las soluciones de seguridad humana sostenibles para enfrentar la Trata (TAVANTI; TUCKER; WANGERIN, 2015, p. 9). Dicho enfoque incluye un análisis de las instituciones estatales de control y equilibrio, así como del nivel de democracia, ya que, desde la perspectiva de la seguridad humana, un estado débil no es capaz de ejercer plenamente su función primaria de protección social individual y, por tanto, es incapaz de cumplir con su obligación internacional de garante de la dignidad humana (TABUSCA, 2015, p. 264).

En definitiva, uno de los dilemas que se ha planteado pone de relieve la urgencia de aplicación de un enfoque de derechos humanos y desarrollo sostenible, sin evadir de la perspectiva de género a la seguridad humana ${ }^{34}$ para

\footnotetext{
${ }_{34}$ "By not asking such questions, even "humanized" approaches to security can "reinforce gender hierarchies that, in turn, diminish women's (and certain men's) real security" (Tickner 2001:62) "Feminist perspectives recognize the broader themes of dominance inherent in gendered constructions of trafficking victims." (...) "While human security could prove to be a useful political tool for antihuman trafficking advocacy, feminists remind us of how easily such utilitarian discourse can be co-
} 
no correr el riesgo de ser apenas utilitarista. A estos efectos vale recordar que la Trata es un crimen transnacional, altamente rentable y que solo podrá ser contrarrestado con éxito a través de la cooperación internacional sensible a sus causas e impacto en la población de origen y destino.

De ahí que afirmemos que la perspectiva la Seguridad Humana sea una herramienta valiosa en el ETP, toda vez que posibilita: un abordaje humanizado que tenga como titular la protección integral de las víctimas y el respeto a sus derechos humanos. El individuo es el centro de atención, significa también crear oportunidades de emancipación y empoderamiento.

opted to be a problem-solving tool losing all critical value especially in the international security arena (Whitworth 2004). Human security similar to human trafficking (see Lobasz 2009) can be constructed in a way that reflects and reinforces unequal power structures, particularly in the form of gendered hierarchies" (KREIDENWEIS y HUDSON, 2015, p.82). 


\section{Referencias Bibliográficas}

ASAMBLEA GENERAL DE NACIONES UNIDAS. Un concepto más amplio de libertad: desarrollo, seguridady derechos humanos para todos. Informe del Secretario General. Resolución A/59/2005, 21 de marzo de 2005.

ASAMBLEA GENERAL DE NACIONES UNIDAS. Seguridad humana. Informe Secretario General. Resolución A/74/701, 8 de marzo de 2010.

BALDWIN, David. "The Concept of Security," Review of International Studies, Vol. 23, no 1, 1997, pp.5-26. Disponible en: http://www.jstor.org/ stable/20097464.

BUZAN, Barry; HANSEN, Lene. The evolution of International Security Studies. Cambridge University Press, 2009.

COMMISSION ON HUMAN SECURITY. Human Security Now. New York, 2003.

CORREADA SILVA, Waldimeiry. ¿Que se rompan los grilletes! La cooperación internacional para la protección de los derechos humanos de las víctimas de trata de personas desde el Consejo de Europa. Revista FACULTAD DE DERECHO Y CIENCIAS POLÍTICAS. Vol. 44, No. 120, 2014. p. 221-269

FRIMAN, H. RICHARD, and SIMON REICH, eds.. 2007. Human Trafficking, Human Security, and the Balkans. Edited by H. RICHARD FRIMAN and SIMON REICH. University of Pittsburgh Press. http://www.jstor.org/ stable/j.ctt5vkfgr.

Instituto Interamericano de Derechos Humanos (IIDH). Seguridad Humana. Disponible en: https://www.iidh.ed.cr/multic/ default_12.aspx?contenidoid=8c1a30 2f-f00e-4f67-b3e68 a3979cf15cd\&Portal=IIDHSeguridad

IONESCU, Magdalena. A Human Security Approach to Anti-trafficking Policies in the EU: Tackling the Structural Sources of Vulnerability. 2007, (pp.211-230) Disponible en: http://www.desk.c.u-tokyo.ac.jp/download/ es_6_Ionesucu.pdf

KREIDENWEIS, Alex; HUDSON, Natalie F. More Than a Crime: Human Trafficking as Human (In)Security. International Studies Perspectives (2015) 16, 67-85.

McFARLANE, S. Neil; KHONG, Yuen Foong. Human Security and the UN: A Critical History. Indiana University Press, 2006.

MORILLAS BASSEDAS, Pol. "Génesis y evolución de la expresión de la seguridad humana. Un repaso histórico", Revista CIDOB d'Afers Internationals, $\mathrm{N}^{\circ}$ 76, 2006-2007, pp. 47-58. 
PATI, Rosa.Human Trafficking: An Issue of Human and National Security, 4 U. Miami Nat'1 Security \& Armed Conflict L. Rev. 29, 2014. Disponible en: http://repository.law.miami.edu/umnsac/vol4/iss2/5

PERAL, Luis. Las amenazas a la seguridad humana y el problema de los medios de acción. Fundación para las Relaciones Internacionales y el Diálogo Exterior (FRIDE) 2005. Documento de Trabajo no. 15

PÉREZ DE ARMIÑO, Karlos (2006-2007), "El concepto y el uso de la seguridad humana: análisis crítico de sus potencialidades y riesgos", Revista CIDOB d'Afers Internationals, N76, pp. 59-77.

PROGRAMA DE LAS NACIONES UNIDAS PARA EL DESARROLLO (PNUD). Informe sobre Desarrollo Humano 1994. México: Fondo de Cultura Económica, 1994.

ROTHSCHILD, Emma (1995), "What is security? The quest for world order", Daedulus, Vol. 124, N 3, Boston. June 1995. Disponible en: http://web. lexis-nexis.com

RUIZ-GIMÉNEZ ARRIETA, Itziar. "El Feminismo y los Estudios Internacionales". In: Revista de Estudios Politicos (Nueva Época), Núm. 108, Abril-Junio 2000, pp. 325-360.

TABUSCA, Silvia Maria. Trafficking in Persons, a Threat to Human Security In The Developed Countries. The Case of European Union In the Era of GlobalizationNational Strategies Observer No.2/Vol.1, 2015. Disponible en: http://ssrn.com/abstract=2689639

TAVANTI, Marco, Charles E. TUCKER, Gregory J. WANGERIN. "AntiHuman Trafficking is at the core of Human Security: An Introduction to the International Journal of Sustainable Human Security Issue on AntiHuman Trafficking." International Journal of Sustainable Human Security (IJSHS). Volume 2, Number 1 (Year 2014-2015), pp. 7-11

THOMAS, Caroline. Global governance, development and human security: exploring the links. Third World Quarterly, Vol. 22, №2, 2001, pp. 159175.

TICKNER, J. ANN. (2001) Gendering World Politics: Issues and Approaches in the Post-Cold War Era. New York: Columbia University Press.

NACIONES UNIDAS. Un concepto más amplio de la libertad: desarrollo, seguridad y derechos humanos para todos. Informe del Secretario General. A/59/2005, 21 de marzo de 2005.

- Seguridad humana. Informe del Secretario General. A/64/701, 8 de marzo de 2010.

\section{INTERNATIONAL COMMISSION ON}

INTERVENTION AND STATE SOVEREIGNTY (ICISS). The Responsability to Protect. Canadá: International Development Research Centre, Diciembre de 2001. 
. 2005 World Summit Outcome. A/60/L.1, 15 de septiembre

de 2005.

- Report of the Secretary-General on the work of the Organization. A/55/1, 30 de agosto de 2000.

. GENERALASSEMBLY, “AnAgenda for Peace: Preventive diplomacy and related matters", A/RES/47/120, de 18 december 1992.

UNITED NATIONS SECURIY COUNCIL. Resolution 827 (1993). S/RES/827 (1993), 25 de mayo de 1993.

. Resolution 955 (1994). S/RES/955 (1994), 8 de noviembre

de 1994.

febrero de 2011.

UNESCO (2005). Promover la Seguridad Humana: Marcos Éticos, Normativos y Educacionales en América Latina y el Caribe. Doc: SHS/ FPH/PHS/2005/PI/H/1. Disponible en: http://unesdoc.unesco.org/ images/0013/001389/138940S.pdf 